\title{
Review of: "Orail Mediated Calcium Influx Improves Sepsis-induced T Lymphocyte Immunosuppression and Acute Organ Dysfunction"
}

\author{
Eun Jeong Park
}

1 Mie University

Potential competing interests: The author(s) declared that no potential competing interests exist.

In this study, the authors sought to elucidate the role of Orail and SOCE in sepsis-induced immune paralysis. A majority of experimental works lacks scientific soundness, although some of data are interesting. Controls in some of data analyzed are missing. Moreover, the explanations for the results as well as figure legends are insufficient in the text. The text requires a grammatical editing by a native speaker. Here are my comments.

The authors tested Th1 and Th2 frequency in CD4 T cells isolated from spleens of septic mice in the context of in-vitro T-cell stimulation (Figure 1). Then, cellularity of Treg and Th17 cells was examined under in-vitro unstimulated conditions (Figure 3)

- But, some critical data are missing. To convince sepsis-mediated Th1 to Th2 shift and Treg/Th17 imbalance, the data of analyses using all four CD4 T cell subtypes (Th1, Th2, Th17, and Treg) under both in-vitro stimulation (anti-CD3/anti-CD28) and un-stimulation conditions need to be included.

- The authors used anti-CD4 and anti-CD25 to detect Treg cells. To ascertain their sepsis-mediated alteration in both function and quantity, subsequent changes in levels of IL-10 and/or TGF-beta need to be shown along with CD4/CD25 levels.

Figure 5 indicates sepsis-induced inhibition of Ca2+-calcineurin-NFAT signaling pathways in splenic CD4 T cells. But, the explanations for the results shown in Figure 5 are not sufficient in the text. The authors stated that nuclear translocation of NFAT1 in the splenic CD4 T cells was inhibited by sepsis in the fluorescent images of Figure 5A. But, the results are not clear. The authors should include better images and more explanations for the sepsis-mediated decrease in the NFAT1 nuclear translocation. How many images and how many cells were used to obtain the results of bar graph of the Figure 5A?

Figure 6 indicates sepsis-induced inhibition of Tg-dependent Ca2+ influx in splenic CD4 T cells. The authors should explain more about the results shown in Figure 6 in the text as well as Figure 6 legend. Are the bar graph in Figure 6 resulted from the levels of fluorescence intensity of R3 gates? What is the "fluorescence intensity increase" in the $Y$ axis? Does it indicate "mean fluorescence intensity"? 
Figure 9 is not visible.

In Figures 10-12, the explanations for this result are not sufficient. What is the control for LV-Orail injection, such as lentiviral vector alone? What is the control for CLP 24h group, such as sham group? Are the LV-Orail group mice also done with CLP? To my understanding, it is imperative to compare between sham and CLP 24h group and between LV-Orai1 overexpression and LV vector only group.

The original full-sized blots of Western blot analyses need to be included in Supplementary Data.

Again, there are too many grammatical errors and typos. The English editing by a native speaker is prerequisite. 